\title{
Velocity Profile Method for Time Varying Resistance in Minimal CVS Models
}

\author{
Bram W Smith†, J. Geoffrey Chase†, Roger I. Nokes $\ddagger$, \\ Geoffrey M. Shaw§and Tim David $\dagger$ \\ $\dagger$ Dept of Mechanical Engineering, University of Canterbury, Christchurch, New \\ Zealand \\ $\ddagger$ Dept of Civil Engineering, University of Canterbury, Christchurch, New Zealand \\ $\S$ Christchurch Hospital Dept of Intensive Care, Christchurch, New Zealand \\ E-mail: g. chase@mech. canterbury .ac.nz
}

\begin{abstract}
.
This paper investigates the fluid dynamics governing arterial flow used in lumped parameter CVS models, particularly near the heart where arteries are large. Assumptions made in applying equations conventionally used in lumped parameter models are investigated, specifically that of constant resistance to flow. The Womersley number is used to show that the effects of time varying resistance must be modeled in the pulsatile flow through the large arteries near the heart. It is shown that the equation commonly used to include inertial effects in fluid flow calculations is inappropriate for including time varying resistance. A method of incorporating time varying resistance into a lumped parameter model is developed that uses the NavierStokes equations to track the velocity profile. Tests on a single chamber model show a $17.5 \%$ difference in cardiac output for a single chamber ventricle model when comparing constant resistance models with the velocity profile tracking method modeling time varying resistance. This increase in precision can be achieved using 20 nodes with only twice the computational time required. The method offers a fluid dynamically and physiologically accurate method of calculating large Womersley number pulsatile fluid flows in large arteries around the heart and valves. The proposed velocity profile tracking method can be easily incorporated into existing lumped parameter CVS models, improving their clinical application by increasing their accuracy.
\end{abstract}

Submitted to: Phys. Med. Biol.

MSC numbers: 92C35,92C50,76Z05,92C10 


\section{Introduction}

Lumped parameter cardiovascular system models offer a relatively simple and computationally inexpensive method of capturing the essential dynamics of the CVS (Burkhoff and Tyberg 1993, Santamore and Burkhoff 1991, Olansen et al 2000, Beyar et al 1987, Ursino 1999, Hardy et al 1982, Sun et al 1997). Finite element methods are the most commonly used alternative, offering extreme resolution at the cost of extreme complexity (Peskin and McQueen 1992, Legrice et al 1997, Smaill and Hunter 1991). When lumped parameter methods sometimes do not capture enough detail there is a need to include some of the complexity and physiologically accurate equations of the finite element approach. Complexity costs computational power and time, and should therefore only be added where significant benefits are obtained over a simpler method. Hence, the addition of complexity to make a lumped parameter model more physiologically accurate must be justified by demonstrating significant changes in model performance.

Typical lumped parameter model equations governing arterial flow rate come in two forms, either including or not including inertial effects. In both cases, resistance to blood flow is typically assumed constant under varying flow velocity and acceleration. Realistically, resistance to flow varies with time for pulsatile flow, and this paper investigates the amount of variation.

The simplest and most common method of modeling blood flow in the CVS is to calculate flow rate as a function of pressure gradient and constant resistance (Hoppensteadt and Peskin 2002, Chung et al 1997, Burkhoff and Tyberg 1993, Vis et al 1997, Santamore and Burkhoff 1991). Other models have included inertial effects as well as a resistance (Olansen et al 2000, Beyar et al 1987, Ursino 1999, Tsitlik et al 1992, Hardy et al 1982, Sun et al 1997, Avanzolini et al 1989, Melchior et al 1992). Often models are based on well-accepted electrical circuit analogies with the advantage that electrical circuit analysis techniques can be used to model the CVS (Ursino 1999, Tsitlik et al 1992, Santamore and Burkhoff 1991, Lu et al 2001, Olansen et al 2000).

A method of including the effect of time varying resistance on flow rate into lumped parameter models is proposed. The method is applied to a simple single chamber model designed to simulate a single ventricular chamber. Models with time varying resistance are then compared with models that assume constant resistance to investigate the differences in performance between them.

\section{Equations Governing Flow-Rate}

Assuming laminar incompressible Newtonian flow, the Navier-Stokes equations in cylindrical-polar co-ordinates may be written as: 


$$
\begin{aligned}
& \frac{\partial \mathrm{u}_{\mathrm{x}}}{\partial \mathrm{t}}+\mathrm{u}_{\mathrm{r}} \frac{\partial \mathrm{u}_{\mathrm{x}}}{\partial \mathrm{r}}+\frac{\mathrm{u}_{\theta}}{\mathrm{r}} \frac{\partial \mathrm{u}_{\mathrm{x}}}{\partial \theta}+\mathrm{u}_{\mathrm{x}} \frac{\partial \mathrm{u}_{\mathrm{x}}}{\partial \mathrm{x}} \\
& =-\frac{1}{\rho} \frac{\partial \mathrm{P}}{\partial \mathrm{x}}+\nu\left[\frac{1}{\mathrm{r}} \frac{\partial}{\partial \mathrm{r}}\left(\mathrm{r} \frac{\partial \mathrm{u}_{\mathrm{x}}}{\partial \mathrm{r}}\right)+\frac{1}{\mathrm{r}^{2}} \frac{\partial^{2} \mathrm{u}_{\mathrm{x}}}{\partial \theta^{2}}+\frac{\partial^{2} \mathrm{u}_{\mathrm{x}}}{\partial \mathrm{x}^{2}}\right]
\end{aligned}
$$

where $\mathrm{u}_{\mathrm{x}}, \mathrm{u}_{\mathrm{r}}$ and $\mathrm{u}_{\theta}$ are the longitudinal, radial and angular velocities respectively, $\mathrm{P}$ is the modified pressure relative to hydrostatic, $\rho$ is the density and $\nu$ is the kinematic viscosity.

The following assumptions are applied:

- The arteries through which blood flows are assumed to be rigid with constant crosssectional area $\left(\frac{\partial \mathrm{r}}{\partial \mathrm{x}}=0\right)$. This assumption fits in with standard windkessel type circuit design involving a rigid pipe and a elastic chamber in series. The rigid tube simulates the fluid dynamics while the elastic chamber simulates the compliance of the artery walls (Tsitlik et al 1992, Melchior et al 1992).

- Laminar axi-symmetric flow is assumed as the tube is rigid with constant radius $\left(\mathrm{u}_{\mathrm{r}}=0, \mathrm{u}_{\theta}=0, \frac{\partial \mathrm{u}_{\mathrm{x}}}{\partial \theta}=0\right)$. Although turbulence can occur around the valves, it takes time to develop, and is assumed not to affect the flow profile significantly.

- The flow is assumed fully developed along the length of the tube meaning the velocity profile is constant with respect to $\mathrm{x}\left(\frac{\partial \mathrm{u}_{\mathrm{x}}}{\partial \mathrm{x}}=0\right)$ (Fung 1993).

- Pressure is assumed constant across the cross-sectional area and the pressure gradient is constant along the length of each section so that the pressure gradient is a function of time only $\left(\frac{\partial \mathrm{P}}{\partial \mathrm{x}}(\mathrm{t})\right)$.

These assumptions enable Equation (1) to be reduced to the following simplified version of the Navier-Stokes equation:

$$
\rho \frac{\partial \mathrm{u}(\mathrm{r}, \mathrm{t})}{\partial \mathrm{t}}=-\frac{\partial \mathrm{P}}{\partial \mathrm{x}}(\mathrm{t})+\frac{\mu}{\mathrm{r}} \frac{\partial}{\partial \mathrm{r}}\left(\mathrm{r} \frac{\partial \mathrm{u}(\mathrm{r}, \mathrm{t})}{\partial \mathrm{r}}\right)
$$

where $\mu$ is the viscosity $(\mu=\nu \rho)$ and $\mathrm{u}(\mathrm{r}, \mathrm{t})$ is the velocity in the $\mathrm{x}$-direction $\left(\mathrm{u}_{\mathrm{x}}(\mathrm{r}, \mathrm{t})\right)$ as a function of radius and time only.

\subsection{Poiseuille's Equation for Flow Rate with Constant Resistance}

If the additional assumption of steady-fully developed flow is applied then Equation (2) reduces to:

$$
0=-\frac{\partial \mathrm{P}}{\partial \mathrm{x}}(\mathrm{t})+\frac{\mu}{\mathrm{r}} \frac{\partial}{\partial \mathrm{r}}\left(\mathrm{r} \frac{\partial \mathrm{u}(\mathrm{r}, \mathrm{t})}{\partial \mathrm{r}}\right)
$$

This equation can be integrated to obtain the flow rate (Q) through a pipe of radius $\left(r_{0}\right)$, as shown in Figure 1, under a pressure gradient $(\partial P / \partial x)$ (White 1991, Fung 1990).

$$
\mathrm{Q}(\mathrm{t})=\frac{\pi \mathrm{r}_{0}^{4}}{8 \mu} \frac{\mathrm{P}_{2}(\mathrm{t})-\mathrm{P}_{1}(\mathrm{t})}{\ell}
$$


with upstream pressure $\left(\mathrm{P}_{1}(\mathrm{t})\right)$, downstream pressure $\left(\mathrm{P}_{2}(\mathrm{t})\right)$ and artery length $(\ell)$. By grouping the constants an equation for resistance is derived.

$$
\mathrm{R}=8 \mu \ell / \pi \mathrm{r}_{0}^{4}
$$

Equation (4) can then be simplified to the electrical analogy commonly used in the literature to calculate the flow rate of blood through arteries and valves (Chung et al 1997, Beyar et al 1987, Burkhoff and Tyberg 1993, Ursino 1999, Olansen et al 2000, Smith et al 2003a,b).

$$
\mathrm{Q}(\mathrm{t})=\frac{\mathrm{P}_{2}(\mathrm{t})-\mathrm{P}_{1}(\mathrm{t})}{\mathrm{R}}
$$

Note that Equation (6) does not include inertial effects or the effects of time varying resistance that influence pulsatile flow.

\subsection{Inclusion of Inertial Effects}

In many parts of the arterial tree, such as the capillaries, the variation in velocity may be minimal, however significant changes in velocity will occur around the heart valves and the flow can no longer be assumed to be steady. Integrating Equation (2) across the cross-sectional area and along the length produces an equation governing the rate of change of flow rate.

$$
\begin{aligned}
& \int_{\mathrm{x}_{1}}^{\mathrm{x}_{2}} \int_{0}^{2 \pi} \int_{0}^{\mathrm{r}_{0}} \rho \frac{\partial \mathrm{u}(\mathrm{r}, \mathrm{t})}{\partial \mathrm{t}} \mathrm{rdrd} \theta \mathrm{dx} \\
& =\int_{\mathrm{x}_{1}}^{\mathrm{x}_{2}} \int_{0}^{2 \pi} \int_{0}^{\mathrm{r}_{0}}\left[-\frac{\partial \mathrm{P}}{\partial \mathrm{x}}(\mathrm{t})+\frac{\mu}{\mathrm{r}} \frac{\partial}{\partial \mathrm{r}}\left(\mathrm{r} \frac{\partial \mathrm{u}(\mathrm{r}, \mathrm{t})}{\partial \mathrm{r}}\right)\right] \mathrm{rdr} \theta \mathrm{dx} \\
& \rho\left(\mathrm{x}_{2}-\mathrm{x}_{1}\right) \frac{\partial \mathrm{Q}(\mathrm{t})}{\partial \mathrm{t}}=\pi \mathrm{r}_{\mathrm{o}}^{2}\left(\mathrm{P}_{1}(\mathrm{t})-\mathrm{P}_{2}(\mathrm{t})\right)+2 \pi \mathrm{r}_{0} \mu\left(\mathrm{x}_{2}-\mathrm{x}_{1}\right) \frac{\partial \mathrm{u}\left(\mathrm{r}_{0}, \mathrm{t}\right)}{\partial \mathrm{r}} \\
& \frac{\rho \ell}{\pi \mathrm{r}_{0}^{2}} \frac{\partial \mathrm{Q}}{\partial \mathrm{t}}=\mathrm{P}_{1}-\mathrm{P}_{2}+\frac{2 \mu \ell}{\mathrm{r}_{0}} \frac{\partial \mathrm{u}}{\partial \mathrm{r}}\left(\mathrm{r}_{0}\right)
\end{aligned}
$$

where the artery length is defined $\ell=\left(\mathrm{x}_{2}-\mathrm{x}_{1}\right)$ and $\frac{\partial \mathrm{u}\left(\mathrm{r}_{0}\right)}{\partial \mathrm{r}}$ is the velocity gradient at the wall of the tube. Equation (9) can then be compared with the equation for flow rate with inertial effects commonly found in the literature (Olansen et al 2000, Beyar et al 1987, Ursino 1999):

$$
\mathrm{LQ}=\mathrm{P}_{1}-\mathrm{P}_{2}-\mathrm{QR}
$$

The derivation of Equation (10) can be found from electrical circuit analogies for an inductor and a resistor in series. 
By comparing Equation (9) with Equation (10) the value of the inductance (L) can be found as a function of the fluid properties, making it a measure of the magnitude of the inertial effects on the fluid per unit cross-sectional area.

$$
\mathrm{L}=\frac{\rho \ell}{\pi \mathrm{r}_{0}^{2}}
$$

However, when comparing the resistance terms from Equations (9) and (10) the following identity is found.

$$
\mathrm{QR}(\mathrm{t})=-\frac{2 \mu \ell}{\mathrm{r}_{0}} \frac{\partial \mathrm{u}}{\partial \mathrm{r}}\left(\mathrm{r}_{0}\right)
$$

The definition in Equation (12) implies that the flow rate (Q) and the boundary velocity gradient $\left(\frac{\partial \mathrm{u}}{\partial \mathrm{r}}\left(\mathrm{r}_{0}\right)\right)$ are related by a constant multiplied by $\mathrm{R}$. If $\mathrm{R}$ is assumed constant, meaning the flow is steady, then this relationship is valid. However, for pulsatile flow with time varying resistance $(\mathrm{R}(\mathrm{t}))$, when the flow rate is zero the boundary velocity gradient is not necessarily zero. Figure 2 shows an example where the flow rate is zero, but the boundary velocity gradient is non-zero. If the boundary velocity gradient at zero flow rate is not necessarily zero, $\mathrm{R}(\mathrm{t})$ will be undefined. This inconsistency shows that Equation (10), which is used to simulate inertial effects in many cases of prior research, is not a suitable governing equation for pulsatile fluid flow with time varying resistance, a more suitable alternative is Equation (9) (Olansen et al 2000, Beyar et al 1987, Ursino 1999, Sun et al 1997).

\subsection{The Womersley Number}

The previous two sections have shown the derivation of three different methods of modeling arterial flow, using either of Equations (6), (9) or (10). Equation (6) would be the preferred choice where possible as it is simple and easy to implement. However, if including inertial effects by using Equations (9) or (10) is going to produce significantly more accurate results, then the increase in complexity is justified.

To investigate which equation should be used to model different types of flow, the Womersley number $(\alpha)$ is used, along with a solution to Equation (2) derived by Womersley. The Womersley number is defined as the ratio of the inertial force to the viscous forces in Equation (2) (Fung 1990).

$$
\alpha^{2}=\frac{\text { transient inertial force }}{\text { viscous force }}=\frac{\omega \mathrm{d}^{2}}{\nu}
$$

where $\omega$ is a characteristic frequency, defined as the heart rate (HR) and $d$ is a characteristic length, defined as the radius of an artery $\left(\mathrm{r}_{0}\right)$. In the CVS the heart rate $(\omega)$ and the kinematic viscosity $(\nu)$ do not vary significantly when compared with the variation in the square of the tube radius. Therefore, frequency and kinematic viscosity can be assumed to be constant and the magnitude of the Womersley number 
in the CVS becomes dependent on radius alone. A Womersley number greater than one $(\alpha>1)$ is considered large and indicates that inertial forces are significant. Flow with a low Womersley number less than one $(\alpha<1)$ is assumed to be only negligibly influenced by inertial effects (Zamir 2000).

The analytical solution derived by Womersley to Equation (2) for flow driven by a sinusoidally varying pressure gradient $\left(\frac{\mathrm{dP}}{\mathrm{dx}}(\mathrm{t})\right)$ is defined (White 1991):

$$
\begin{aligned}
& \frac{d P}{d x}(t)=-d P_{a m p} \cdot e^{i \omega t} \\
& u(r, t)=d P_{a m p} \frac{i}{\rho \omega}\left[1-\frac{J_{0}(r \sqrt{-i \omega / \nu})}{J_{0}\left(\mathrm{r}_{0} \sqrt{-i \omega / \nu}\right)}\right]
\end{aligned}
$$

where $\mathrm{dP}_{\mathrm{amp}}$ represents the amplitude of the variation in the pressure gradient, $\omega$ represents the frequency of oscillation, and $J_{0}$ represents a Bessel function of order zero. Values employed for these constants are outlined in Table 1. The amplitude of pressure gradient variation is set as $5 \mathrm{mmHg}$ to approximate the pressure drop in the aorta.

Two different flow cases are modeled for flow through a tube and the profiles of the variation in flow rate and boundary velocity gradient are determined numerically. The first case is flow through a small radius with a low Womersley number $(\alpha=0.009)$ representing the peripheral circulation, such as flow through the capillaries. The second case simulates a large radius, indicating a large Womersley number $(\alpha=55)$, such as in the larger arteries and veins near the heart. Figure 3 shows the results where the top graph plots the variation in pressure gradient with time. The middle graph plots both the flow rate $(\mathrm{Q})$ and the boundary velocity gradient $\left(\frac{\partial \mathrm{u}}{\partial \mathrm{r}}\left(\mathrm{r}_{0}\right)\right)$ overlaid for a small Womersley number. It is shown that the boundary velocity gradient is directly in phase with the flow rate for small Womersley number flow. The bottom graph shows the same information for a large Womersley number, where the flow rate and the boundary velocity gradient are considerably out of phase.

In the arteries and veins close to the heart the Womersley number is large, inertial forces are significant, and they must be included in fluid flow calculations. For large Womersley number flow inertial effects on the fluid cause the flow rate to significantly lag behind the pressure gradient as shown in Figure 3. Additionally, the boundary velocity gradient is shown to be out of phase with the flow rate. By relating this phase lag to Equation (12), it can be seen that resistance must vary with time to accommodate the out of phase variations in $\mathrm{Q}$ and $\partial \mathrm{u} / \partial \mathrm{r}\left(\mathrm{r}_{0}\right)$. Equation (10) assumes constant resistance and is therefore not suitable for modeling this flow, and Equation (9) should be used as a more accurate alternative based directly on the Navier-Stokes equation and fluid dynamic principles.

Therefore, for large arteries with a high Womersley number flow, both inertial effects and the effects of time varying resistance must be simulated by using Equation (9). 
When simulating flow through the smaller vessels in the peripheral circulation, Equation (6) is more suitable as it requires significantly less computational time. By using a suitable combination of these two equations, a more physiologically accurate closed loop model of the CVS can be constructed and solved in optimum time.

\section{Single Chamber CVS Model}

To demonstrate and quantify the relative performance of Equations (9) and (10), a single chamber model is used, as shown in Figure 4. The single elastic chamber is modeled as a resistor, an inductor and a "valve" at both the inlet and outlet to simulate resistance, inertial effects and the cardiac valves. Such chambers have been successfully used to model the dynamics of a single ventricle with constant upstream and downstream pressures, and can be combined to create larger models with many chambers (Beyar et al 1987, Olansen et al 2000, Santamore and Burkhoff 1991, Smith et al 2003a,b).

The present model uses a time varying elastance $(\mathrm{E}(\mathrm{t}))$ to simulate the cardiac muscle activation. The upper and lower limits of the elastance are defined by the end-systolic pressure-volume relationship (ESPVR) and end-diastolic pressure-volume relationship (EDPVR) respectively (Chung et al 1997). By combining the ESPVR and EDPVR an equation for the pressure in a cardiac chamber as a function of time and chamber volume is found (Smith et al 2003a, Chung et al 1997, Santamore and Burkhoff 1991, Beyar et al 1987).

$$
\mathrm{P}_{2}(\mathrm{~V}, \mathrm{t})=\mathrm{e}(\mathrm{t}) \mathrm{E}_{\mathrm{es}}\left(\mathrm{V}-\mathrm{V}_{\mathrm{d}}\right)+(1-\mathrm{e}(\mathrm{t})) \mathrm{A}\left(\mathrm{e}^{\lambda\left(\mathrm{V}-\mathrm{V}_{\mathrm{o}}\right)}-1\right)
$$

where $\mathrm{e}(\mathrm{t})$ is a driver function that varies in a user defined profile between 0 and 1 . The profile of $\mathrm{e}(\mathrm{t})$ used in this case is defined:

$$
\mathrm{e}(\mathrm{t})=\sum_{\mathrm{i}=1}^{\mathrm{N}} \mathrm{A}_{\mathrm{i}} \mathrm{e}^{-\mathrm{B}_{\mathrm{i}}\left(\mathrm{t}-\mathrm{C}_{\mathrm{i}}\right)^{2}}
$$

where $A_{i}, B_{i}, C_{i}$ and $N$ are parameters that determine the shape of the driver profile. In this case, values of $A_{1}=1, B_{1}=80, C_{1}=0.27$ and $\mathrm{N}=1$ are used, although more complex shapes can be used (Chung et al 1997).

Using Equation (16) the pressure in the chamber is calculated as a function of time and volume, and the pressure gradient between the inlet and outlet can be determined. With the pressure gradient known, either of Equations (6), (9) or (10) can be used to determine the flow rate in and out of the chamber. The rate of change of volume is calculated from the difference in flow rates.

Values of the constants used in single chamber model simulations are shown in Table 1. Blood properties are taken from Guyton (1991) and the heart rate is set to 80 beats/min. Artery properties are approximated based on dimensions found in the literature, with the artery length adjusted to produce reasonable results (Chung et al 
1997, Smith et al 2003a,b). Chamber properties are optimized values initially obtained from other models in the literature for a human (Chung et al 1997, Burkhoff and Tyberg 1993, Ursino 1999, Smith et al 2003a,b).

\subsection{Simulating the Single Chamber Model}

The single chamber model is numerically integrated using Matlab's ode15s ODE solver. Conventionally used models use either Equation (6) or Equation (10) as the governing equations for flow rate. Where Equation (6) is used, the volume of the chamber is the only state variable $(\underline{x}=[V])$. However, if Equation (10) is used, then volume as well as flow rate in and out are all state variables in the state vector $\left(\underline{x}=\left[\mathrm{V}, \mathrm{Q}_{\mathrm{in}}, \mathrm{Q}_{\text {out }}\right]\right)$.

Models with greater numbers of chambers and complexity are solved the same way by simply adding the volume of every elastic chamber to the state vector. Where inertial effects are included in flow rate calculations, the flow rates are also added as state variables. The result is a numerically stable and physiologically accurate model of basic CVS dynamics (Smith et al 2003a,b).

Valve closure is assumed to occur when net flow rate goes to zero. Research shows that in fact a small amount of reversing flow occurs just before valve closure, but this flow is negligible and ignored in this model (Bellhouse and Talbot 1969). The velocity profile at zero net flow rate is shown in Figure 2, with the flow moving forward along the central axis of the tube and reversing flow around the outside of the tube.

\subsection{Tracking the Velocity Profile}

It has been shown that Equation (9) should be used when trying to model the effects of time varying resistance and inertial effects. The question is how to add this equation into a lumped parameter model to replace Equation (10). This paper presents a method where instead of just determining the flow rate $(\mathrm{Q})$, the velocity profile vector $(\underline{u})$ is added as a series of state variables in the state vector. In this way, not just the flow rate will be determined, but also the variations in the velocity profile, enabling the boundary velocity gradient and resistance to be determined at any given time.

The velocity profile vector calculated is a discrete representation of the velocity profile, dividing the radius into equally spaced nodes. Instead of using Equation (9), Equation (2) is now used since the velocity profile, and not the flow rate, is obtained at each time step. At each iteration of the ODE solver, the rate of change of velocity is calculated at each node using Equation (2). A non-slip boundary condition is applied. Thus, where Equation (10) is often used to calculate the rate of change of flow rate, Equation (9) would now be used to calculate the rate of change of the velocity vector.

Using this method, the state vector is of the form:

$$
\underline{\mathrm{x}}=[\mathrm{V}, \underbrace{\mathrm{u}_{\text {in.1 }}, \mathrm{u}_{\text {in.2 }}, \ldots, \mathrm{u}_{\text {in.i. }}}_{\underline{\mathrm{u}}_{\text {in }}}, \underbrace{\mathrm{u}_{\text {out.1 }}, \mathrm{u}_{\text {out.2 }}, \ldots, \mathrm{u}_{\text {out.i }}}_{\underline{\mathrm{u}}_{\text {out }}}]
$$


where $\underline{u}_{\text {in }}$ and $\underline{u}_{\text {out }}$ are the velocity profile in and out of the chamber respectively. The following steps are taken at each iteration of the ODE solver:

- The chamber volume and the velocity profiles are passed to the ODE function as state variables.

- The chamber pressure is calculated from the volume using Equation (16).

- Equation (2) is used to find the rate of change of the velocity profile.

- The velocity profiles are numerically integrated to calculate the flow rates.

$$
\mathrm{Q}=\int_{0}^{2 \pi} \int_{0}^{\mathrm{r}_{0}} \mathrm{u}(\mathrm{r}, \mathrm{t}) \mathrm{rdrd} \theta
$$

- The rate of change of chamber volume is calculated from the flow rate in less the flow rate out.

- The time derivative of the state vector is passed back to the ODE solver including the rate of change of volume and the rate of change of the velocity profiles.

Thus, time varying resistance and inertial effects are easily included in a simple lumped parameter CVS model. The actual values of inertia (L) and time varying resistance $(\mathrm{R}(\mathrm{t}))$ are not directly calculated in the manner of conventional lumped parameter CVS models. Instead, this method of tracking the velocity profile automatically incorporates these effects.

\subsection{Non-Dimensionalisation}

The magnitude of the variables used in Equation (2) vary significantly, which will contribute to an accumulation of computational error. For example, the magnitude of the volume is on the order of milliliters $\left(10^{-6} \mathrm{~m}^{3}\right)$ and pressure is on the order of $\mathrm{mmHg}\left(10^{2} \mathrm{~N} / \mathrm{m}^{2}\right)$, so that there is an order of $10^{8}$ difference in these numbers. To avoid accumulation of machine error, the equations were non-dimensionalized before being applied to the model.

Non-dimensional values are labeled with a star $\left(^{*}\right)$ so the non-dimensional form of a dimensional variable $\mathrm{x}$ is $\mathrm{x}^{*}$, which is related to $\mathrm{x}$ by it's characteristic value labeled $\overline{\mathrm{x}}$. The characteristic flow rate is defined:

$$
\overline{\mathrm{Q}}=\frac{\pi \overline{\mathrm{r}}^{4}}{8 \mu} \frac{\overline{\mathrm{dP}}}{\mathrm{dx}}
$$

where the characteristic pressure gradient is defined:

$$
\frac{\overline{\mathrm{dP}}}{\mathrm{dx}}=\frac{\overline{\mathrm{P}_{2}-\mathrm{P}_{1}}}{\bar{\ell}}
$$

The characteristic velocity is set as the characteristic flow rate divided by the cross sectional area.

$$
\overline{\mathrm{u}}=\frac{\overline{\mathrm{Q}}}{\pi \overline{\mathrm{r}}^{2}}=\frac{\overline{\mathrm{r}}^{2}}{8 \mu} \frac{\overline{\mathrm{dP}}}{\mathrm{dx}}
$$


The non-dimensional form of the equation for the rate of change of the velocity vector can also be found from Equation (2).

$$
\frac{\partial \mathrm{u}^{*}\left(\mathrm{r}^{*}, \mathrm{t}^{*}\right)}{\partial \mathrm{t}^{*}}=\frac{480 \nu}{\overline{\mathrm{r}}^{2} \mathrm{HR}}\left(-\frac{\partial \mathrm{P}^{*}}{\partial \mathrm{x}}\left(\mathrm{t}^{*}\right)+\frac{1}{8}\left(\frac{\partial^{2} \mathrm{u}^{*}\left(\mathrm{r}^{*}, \mathrm{t}^{*}\right)}{\partial \mathrm{r}^{* 2}}+\frac{1}{\mathrm{r}^{*}} \frac{\partial \mathrm{u}^{*}\left(\mathrm{r}^{*}, \mathrm{t}^{*}\right)}{\partial \mathrm{r}^{*}}\right)\right)
$$

Note that the term $\nu /\left(\overline{\mathrm{r}}^{2} \mathrm{HR}\right)$ is equal to the inverse of the Womersley number squared $\left(1 / \alpha^{2}\right)$.

\section{Results}

For the velocity profile method, nodes at which velocity is calculated are spaced equally along the radius. Before carrying out simulations using the velocity profile method, the number of nodes required to gain accurate results must be found. Using many nodes makes the calculations more accurate, but lengthens the time required to simulate the model. Figure 5 shows a plot of the percentage error of the stroke volume for models using different numbers of radial nodes. The percentage error is relative to the stroke volume at 150 nodes, where the solution has converged and error is negligible. From Figure 5 the number of nodes required can be determined based on the allowable error. For all simulations in this paper using the velocity profile method, 100 nodes were used as this number produces minimal error, although fewer nodes would still produce good results with less computation time.

To verify the velocity profile method, using Equation (2), against the constant resistance method, using Equation (10), inertial effects are minimized in both and the results compared. Inertial effects are minimized by setting the density $(\rho)$ of the blood to a very low value of $1 \mathrm{~kg} / \mathrm{m}^{3}$. Substituting $\nu=\mu / \rho$ into Equation (13), it can be seen that this density decrease will cause a significant decrease in Womersley number, and inertial effects will be negligible. For the constant resistance method, values of $\mathrm{R}$ and $\mathrm{L}$ are calculated using Equation (5) and Equation (11) respectively. In both cases, making the inertial effects negligible means that there is now much less restriction on the rate of change of velocity and the velocity profile develops almost instantly to that defined by Poiseuille (White 1991).

Figure 6 shows the results of each method in a PV diagram. There is virtually no difference in performance between the two. This result verifies that the velocity profile method performs in the same way as the constant resistance method under very low inertia conditions as expected since density, and consequently inertial effects, have been made negligible.

The models are simulated again with the density reset to a normal blood density of $1050 \mathrm{~kg} / \mathrm{m}^{3}$, from Table 1 . Figure 7 compares the performance of the two models. Figure 8 plots the same simulation results as Figure 7 but in a PV diagram. It is found that the velocity profile method results in an approximately $17.5 \%$ decrease in stroke volume, and thus cardiac output. This decrease is a significant change in the cardiac 
performance of the model resulting from using the more physiologically accurate velocity profile method over the widely used constant resistance assumption.

The relative computational speed of the constant resistance method compared with the velocity profile tracking method was found by measuring the computational time required for each method. When 100 nodes are used in the velocity profile method, it took 10 times longer to solve than the constant resistance method. When 20 nodes are used then the solution took only twice as long to solve. Therefore, the increase in cost is an approximately number of nodes divided by 10 times increase in solution time.

\section{Conclusions}

The electrical circuit analogy equations governing flow rate traditionally used in lumped parameter CVS models are investigated and compared with standard fluid dynamics principles and equations. Key assumptions made in the derivation of these equations are outlined and their relevance discussed, particularly the assumption of constant resistance under varying flow rates. Womersley number analysis shows that for large arteries around the heart, inertial effects and time varying resistance must be included in equations governing pulsatile flow. The electrical analogy equation most commonly used to calculate flow rate with inertial effects is shown to be inadequate in simulating large Womersley number flow. As a result, a method for calculating and incorporating time varying resistance into lumped-parameter models is proposed. This method involves tracking the velocity profile, instead of just the flow rates, using a non-dimensionalized governing equation derived from the Navier-Stokes equation. Tests are carried out on a single chamber CVS model comparing the performance of commonly used constant resistance methods with the proposed method of tracking the velocity profile. A $17.5 \%$ difference in cardiac output for pulsatile flow in larger arteries is found, justifying the inclusion of the more physiologically accurate velocity profile tracking method. Adequate precision is achieved when using 20 nodes, which takes approximately twice as long to solve as a constant resistance method. The velocity profile tracking method is presented as a more fluid dynamically, and therefore physiologically, accurate method for simulating pulsatile blood flow in large arteries near the heart. The increased computational effort required to solve a model using this method is justified by the significant change in model output dynamics. 


\section{Figure captions}

Figure 1. Flow through a rigid pipe of constant cross section.

Figure 2. The velocity profile at zero flow rate.

Figure 3. Sinusodially varying pressure gradient driving flow (top), variations in $\partial \mathrm{u} / \partial \mathrm{r}\left(\mathrm{r}_{0}\right)$ and $\mathrm{Q}$ for small Womersley number flow, $\alpha=0.009$, (middle) and large Womersley number flow, $\alpha=55$ (bottom).

Figure 4. The single chamber model.

Figure 5. Percentage error of stroke volume versus number of nodes on radius.

Figure 6. Velocity profile method (VProf) and constant resistance method (CR) results overlaid with inertial effects assumed to be negligible.

Figure 7. Velocity profile method (VProf) and constant resistance (CR) results overlaid with density $=1050 \mathrm{~kg} / \mathrm{m}^{3}$.

Figure 8. Velocity profile method results (VProf) overlaid on constant resistance results $(\mathrm{CR})$ on a PV diagram. 


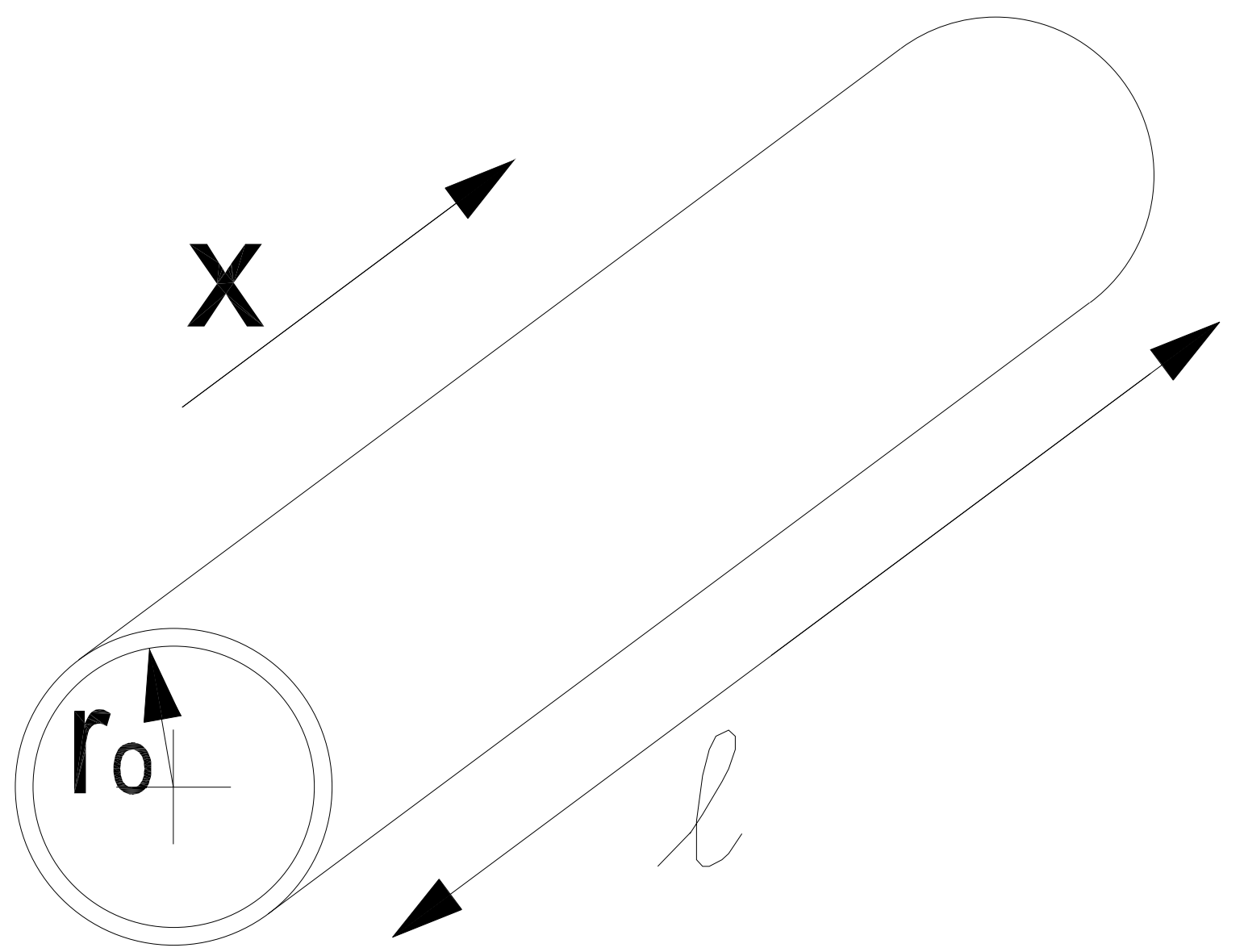

Figure 1. Flow through a rigid pipe of constant cross section 


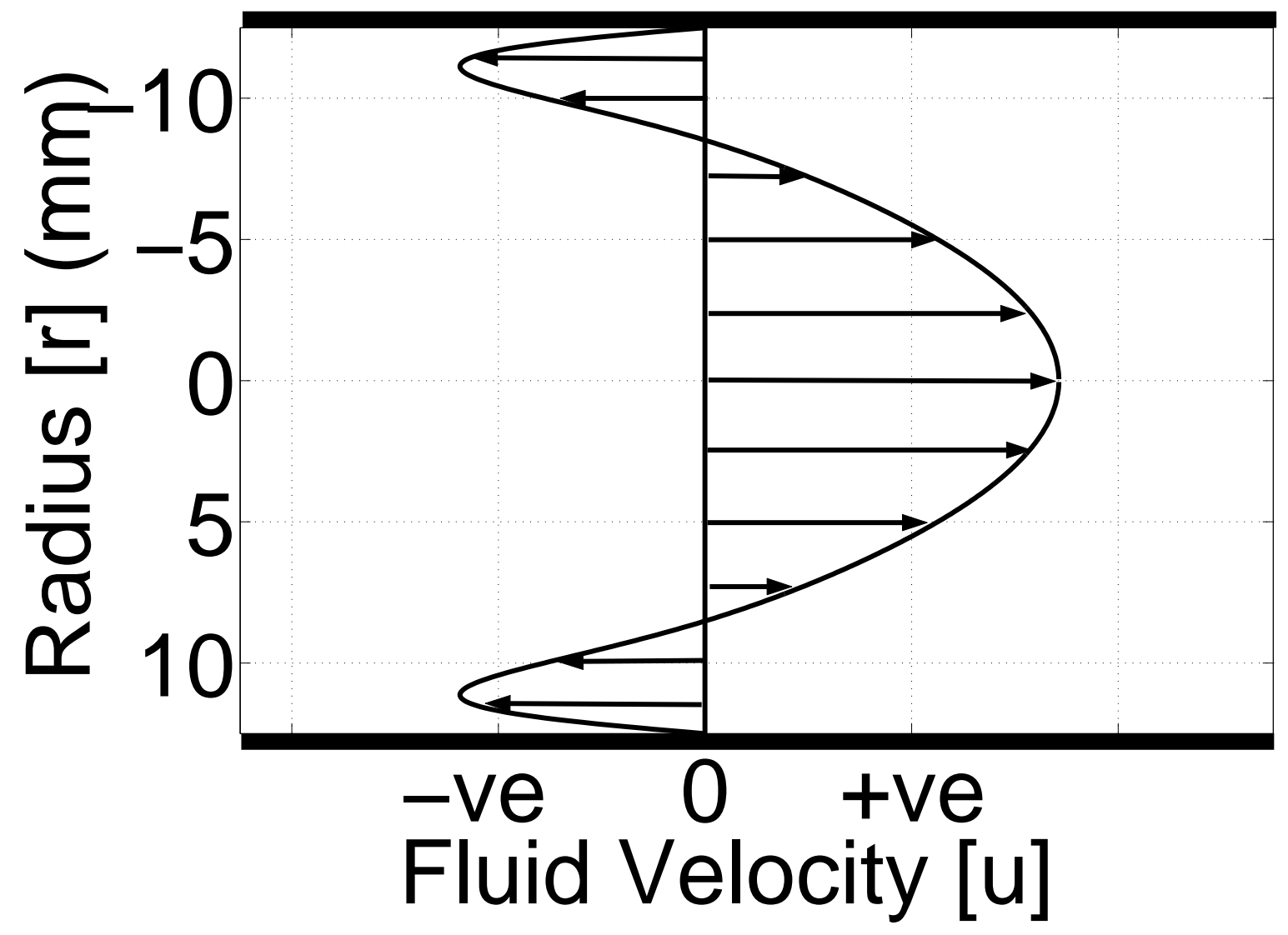

Figure 2. The velocity profile at zero flow rate. 

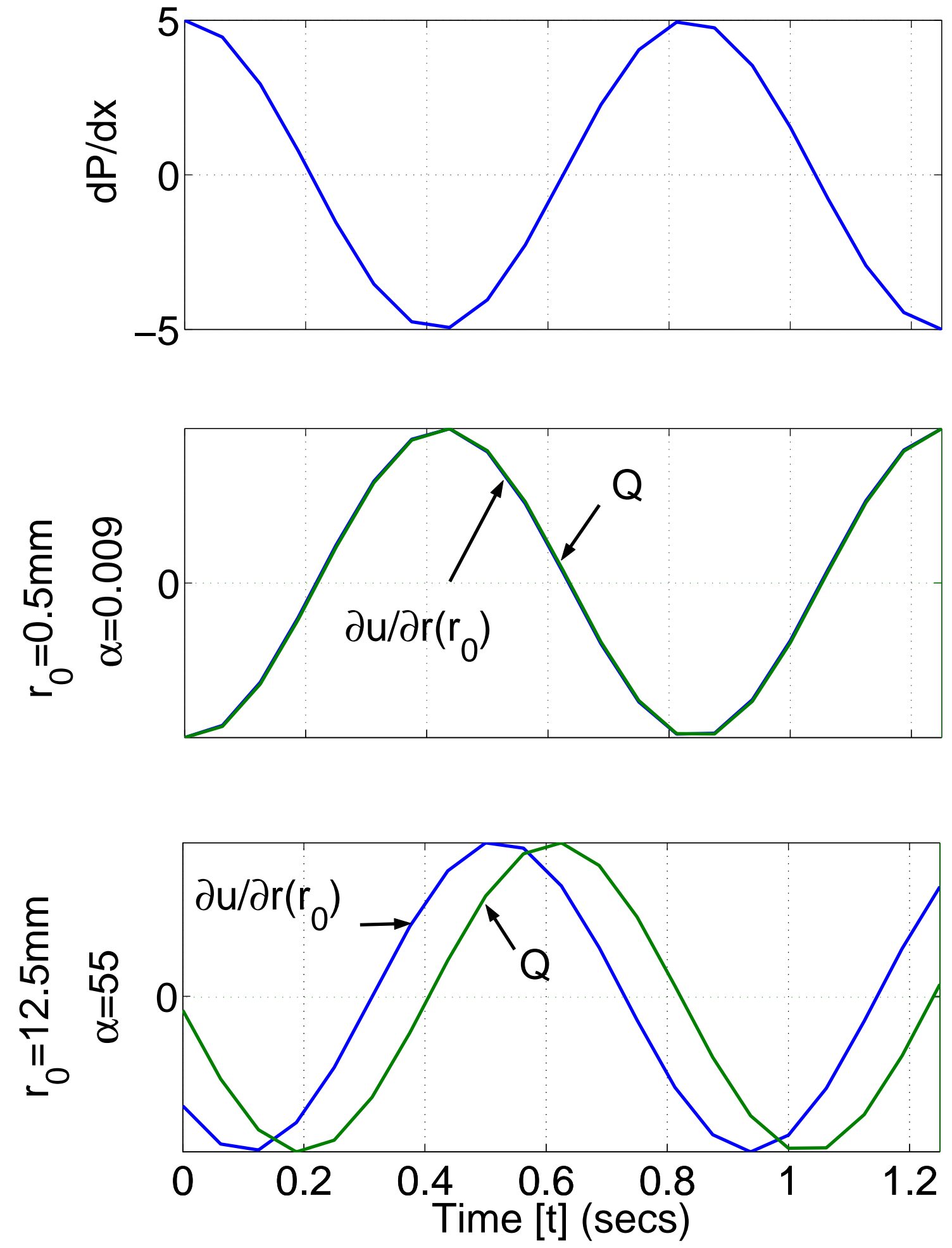

Figure 3. Sinusodially varying pressure gradient driving flow (top), variations in $\partial \mathrm{u} / \partial \mathrm{r}\left(\mathrm{r}_{0}\right)$ and $\mathrm{Q}$ for small Womersley number flow, $\alpha=0.009$, (middle) and large Womersley number flow, $\alpha=55$ (bottom) 


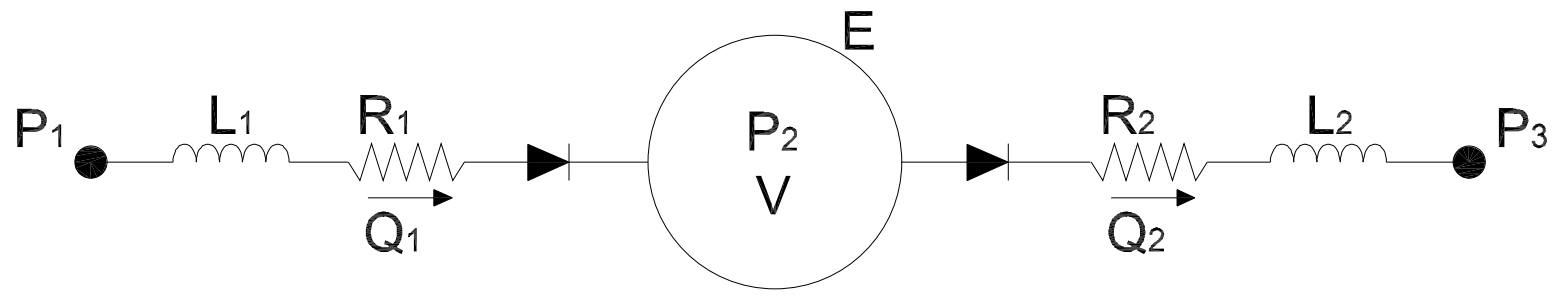

Figure 4. The single chamber model. 


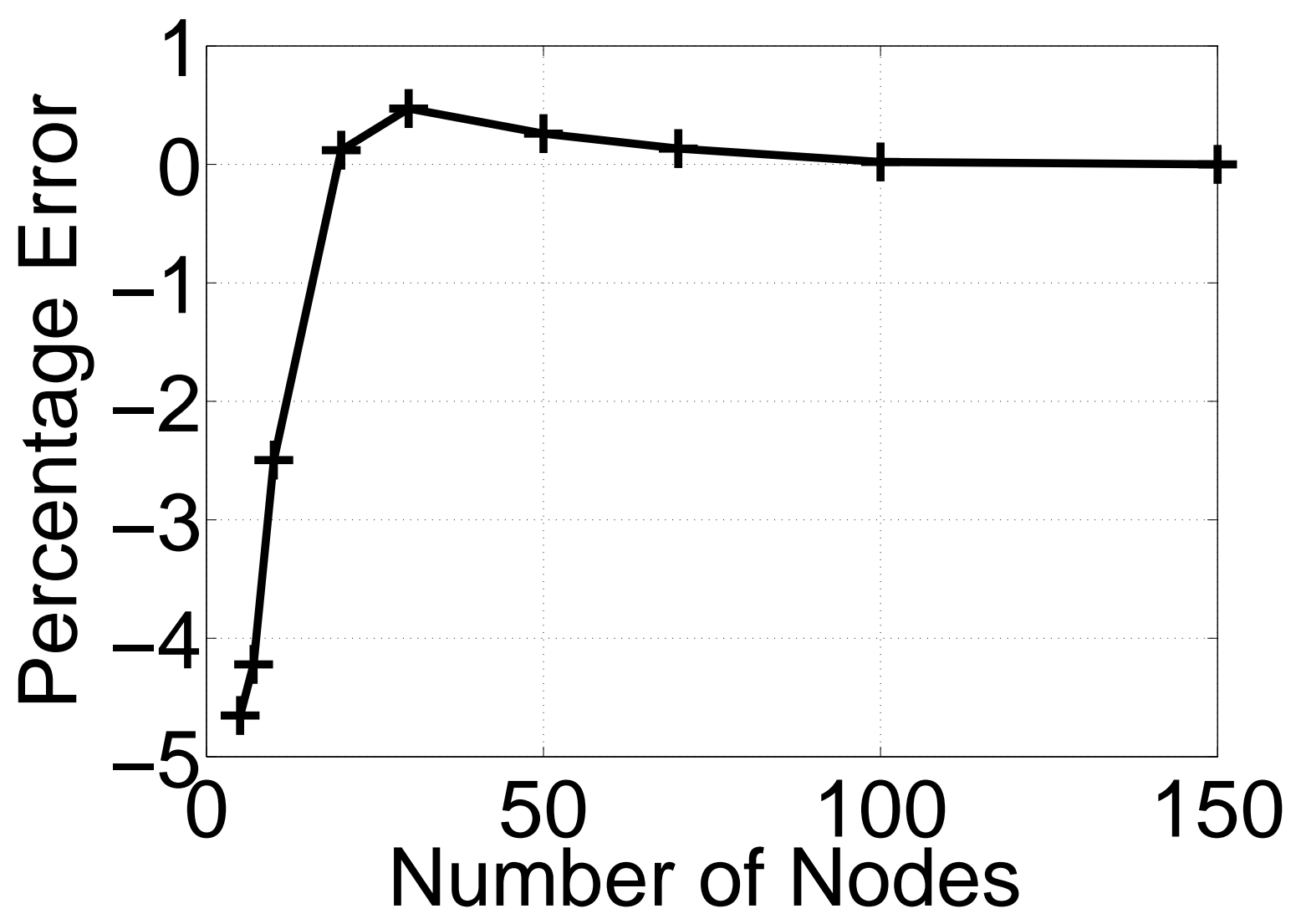

Figure 5. Percentage error of stroke volume versus number of nodes on radius. 


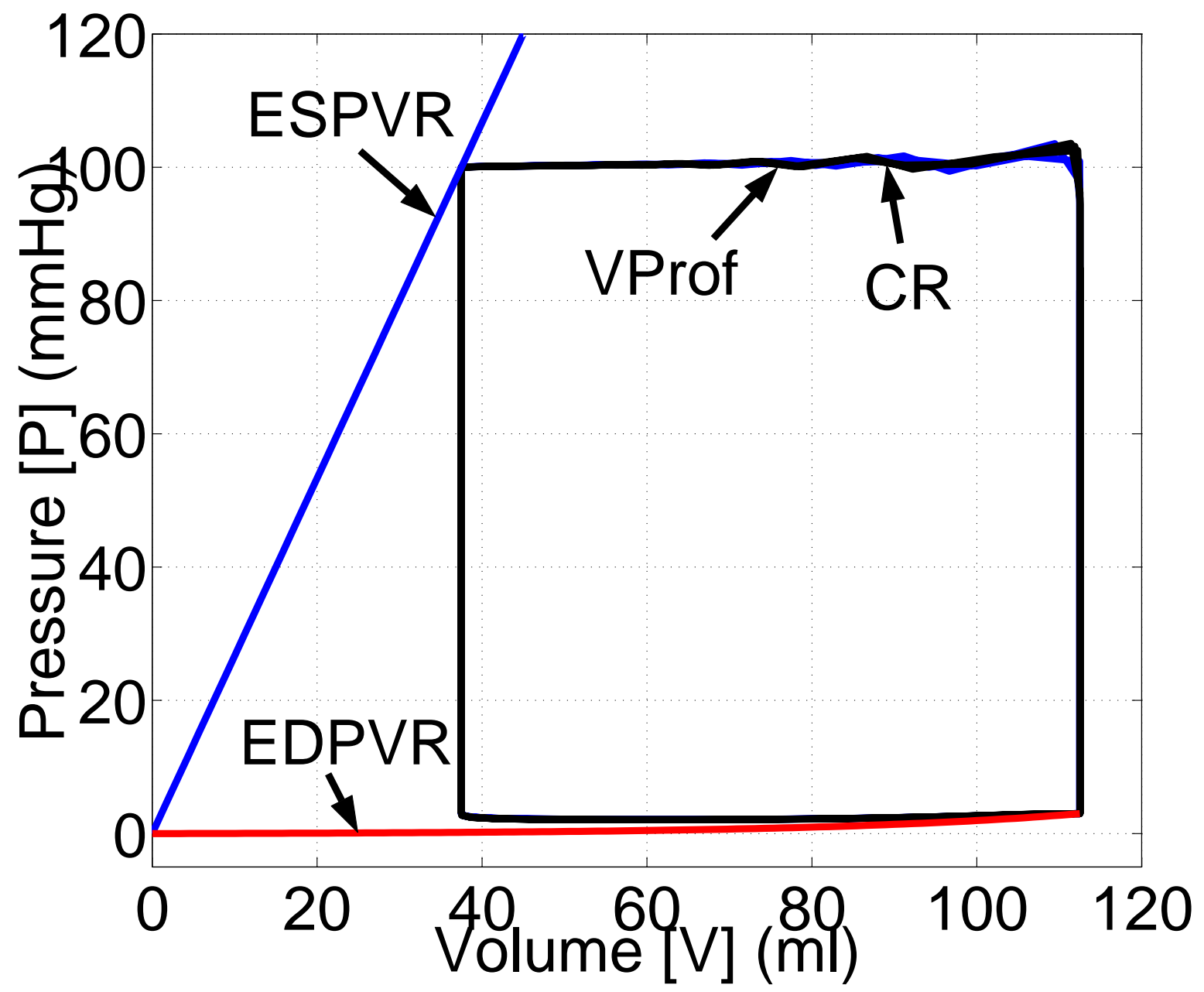

Figure 6. Velocity profile method (VProf) and constant resistance method (CR) results overlaid with inertial effects assumed to be negligible. 

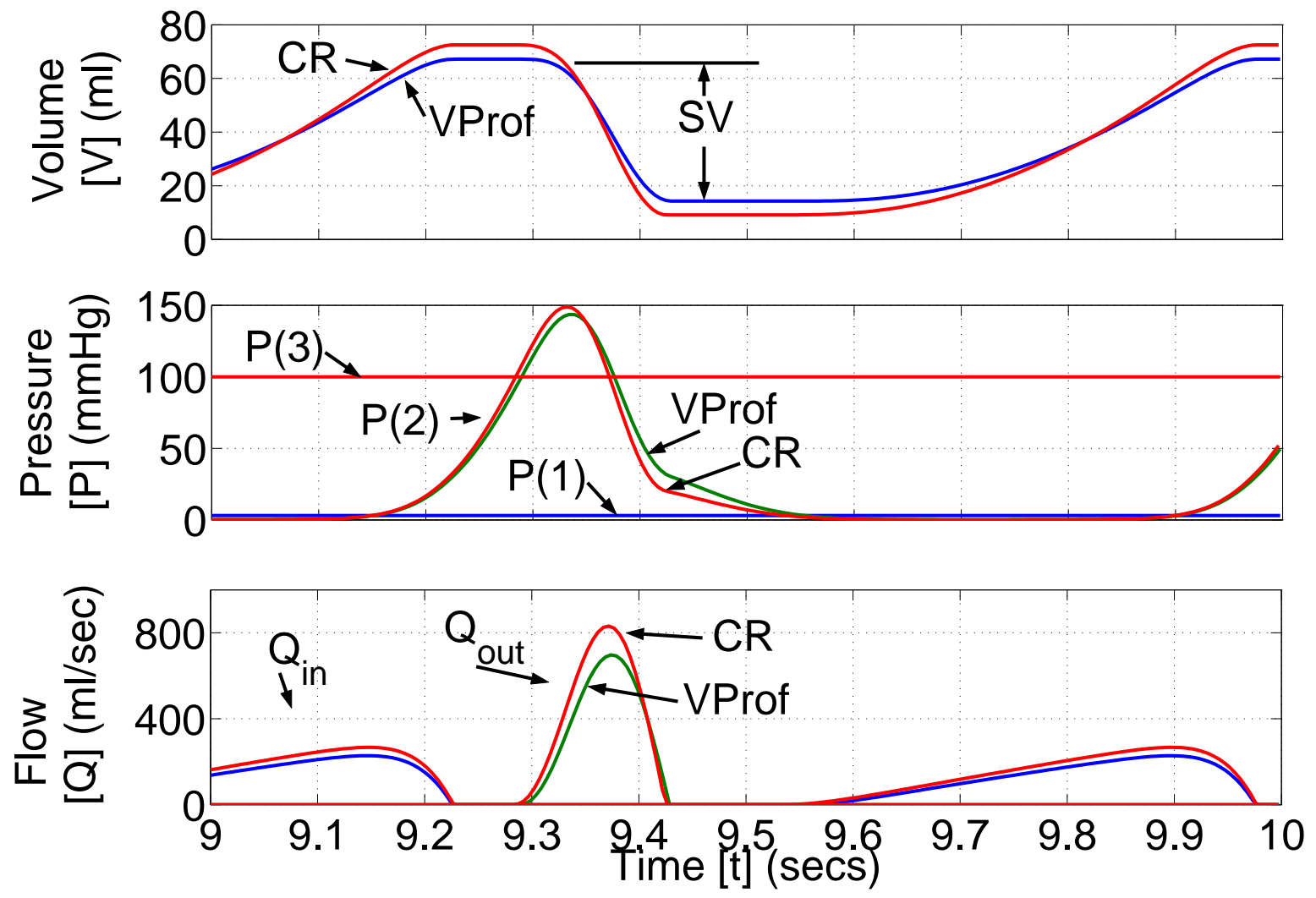

Figure 7. Velocity profile method (VProf) and constant resistance (CR) results overlaid with density $=1050 \mathrm{~kg} / \mathrm{m}^{3}$. 


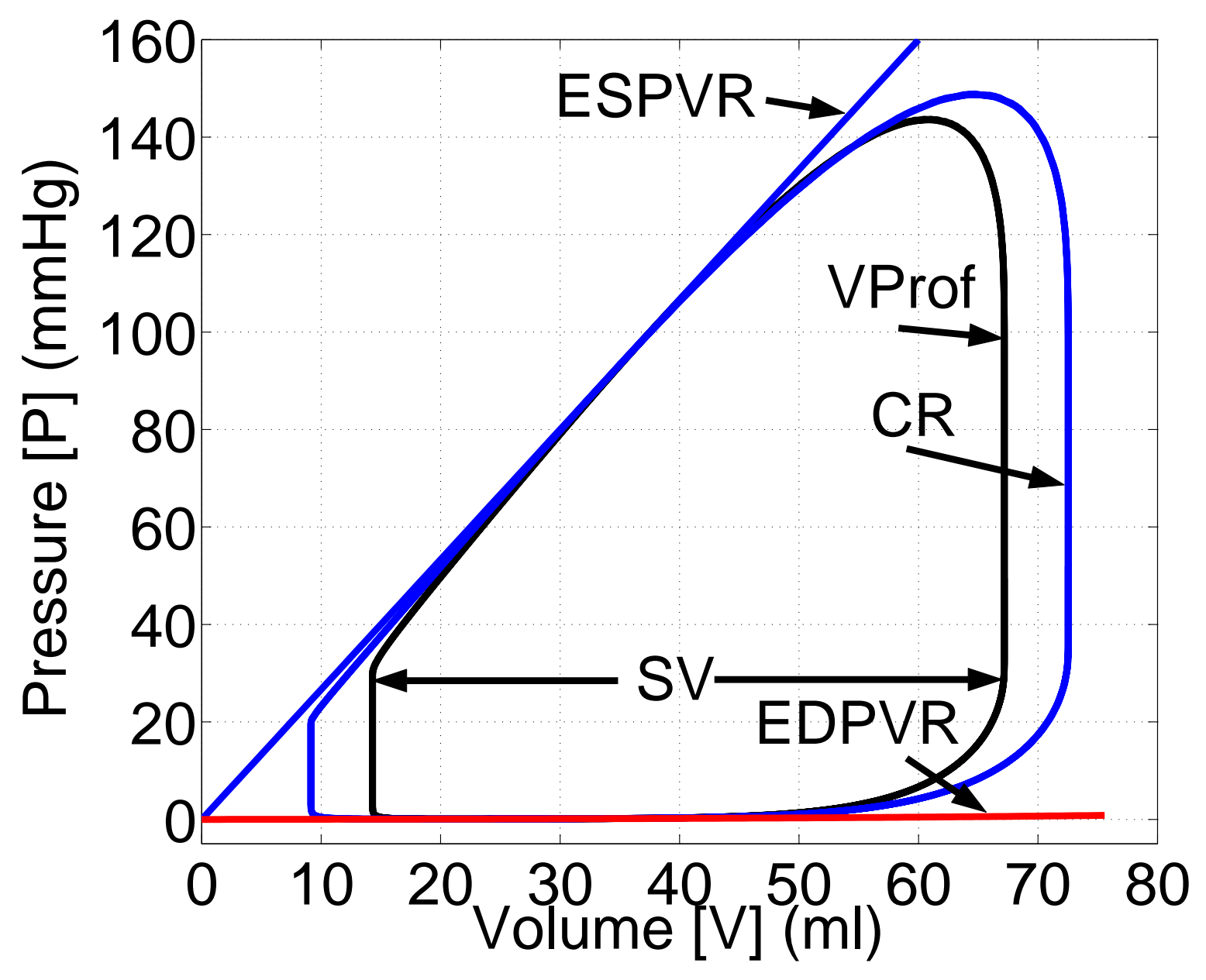

Figure 8. Velocity profile method results (VProf) overlaid on constant resistance results $(\mathrm{CR})$ on a $\mathrm{PV}$ diagram. 
Table 1. Constants used in single-chamber simulation

\begin{tabular}{lll}
\hline Description & Symbol & Value \\
\hline Blood properties: & & \\
\hline Blood density & $\rho$ & $1050 \mathrm{~kg} / \mathrm{m}^{3}$ \\
Blood Viscosity & $\mu$ & $0.004 \mathrm{Ns} / \mathrm{m}^{2}$ \\
Blood Kinematic Viscosity & $\nu$ & $3.8 \times 10^{-6} \mathrm{~m}^{2} / \mathrm{s}$ \\
\hline Artery Properties: & & \\
\hline Internal Artery Radius & $r_{0}$ & $0.0125 \mathrm{~m}$ \\
Artery Length & $\ell$ & $0.2 \mathrm{~m}$ \\
\hline Chamber properties: & & \\
\hline Chamber Elastance & $E_{e s}$ & $3.56 \times 10^{8} \mathrm{~N} / \mathrm{m}^{5}$ \\
EDPVR Volume Cross-over & $V_{0}$ & $0 \mathrm{~m}^{3}$ \\
ESPVR Volume Cross-over & $V_{d}$ & $0 \mathrm{~m}^{3}$ \\
Constant & $\lambda$ & $33000 \mathrm{~m}^{-3}$ \\
Heart Rate & $\omega$ & $1.33 \mathrm{beats} / \mathrm{sec}$ \\
Constant & $\mathrm{A}$ & $10 \mathrm{~N} / \mathrm{m}^{2}$ \\
\hline
\end{tabular}

